\title{
About t-norms on type-2 fuzzy sets
}

\author{
Pablo Hernández ${ }^{1}$ Susana Cubillo ${ }^{2}$ Carmen Torres ${ }^{2}$ \\ ${ }^{1}$ Departamento de Matemática y Física, Univ. Nacional Experimental del Táchira, \\ San Cristóbal, Táchira, Venezuela, phernandezv@unet.edu.ve \\ ${ }^{2}$ Departamento de Matemática Aplicada, Univ. Politécnica de Madrid, \\ 28660 Boadilla del Monte, Madrid, España, \{scubillo,ctorres\}@fi.upm.es
}

\begin{abstract}
Walker et al. ([18], [19]) defined two families of binary operations on $\mathbf{M}$ (set of functions of $[0,1]$ in $[0,1])$, and they determined that, under certain conditions, those operations are t-norms (triangular norm) or t-conorms on $\mathbf{L}$ (all the normal and convex functions of $\mathbf{M}$ ). We define binary operations on $\mathbf{M}$, more general than those given by Walker et al., and we study many properties of these general operations that allow us to deduce new t-norms and t-conorms on both $\mathbf{L}$, and $\mathbf{M}$.
\end{abstract}

Keywords: Functions of $[0,1]$ in $[0,1]$, normal and convex functions, t-norm and t-conorm.

\section{Introduction}

Type-2 fuzzy sets (T2FSs) were introduced by L.A. Zadeh in 1975 [21], as an extension of type-1 fuzzy sets (FSs). Whereas an element's degree of membership in type-1 fuzzy sets is determined by a value in the interval $[0,1]$, an element's degree of membership in a T2FS is a fuzzy set in $[0,1]$. Then, a T2FS is determined by a membership function $\mu: X \rightarrow[0,1]^{[0,1]}$, where $\mathbf{M}=[0,1]^{[0,1]}$ is the set of functions of $[0,1]$ in $[0,1]$ (see $[10,12,13,18]$ ). In this paper, some general results in T2FSs with degrees of membership in $\mathbf{M}$ will be obtained, as well as particular results for T2FSs with degrees of membership in the subset $\mathbf{L}$ of normal and convex functions of $\mathbf{M}$.

Triangular norms (t-norms) were introduced by Menger [11], and later, B. Schweizer and A. Sklar in $[16,15]$ gave the axiomatic currently used to define t-norm. Because of the close connection between the theory of fuzzy sets and order theory (see, eg, [6]), several authors have studied t-norms on bounded partially ordered sets (bounded posets). In this direction, in [4] and [3] the notion of t-norm was generalized for bounded posets. More, in [14] the extension of t-norm on bounded lattices was considered, establishing the axioms (hereinafter called "basic" axioms), that match those given by [4] and [3].

These definitions were also extended in [5] to interval valued fuzzy sets (IVFSs), but other restrictions or properties were added to the "basic" axioms, establishing the "restrictive" axioms. Later, in $[18,19]$ the authors extended the "restrictive" axioms to T2FSs, and presented two families of binary operations on $\mathbf{M}$, determining that, under certain conditions, the operations are t-norms or tconorms on $\mathbf{L}$. In this paper, we propose two families of binary operations on $\mathbf{M}$, more general than those presented in $[18,19]$, and we analyze, among other properties, in which conditions these families satisfy each of the "restrictive" axioms on $\mathbf{L}$ or on M. In particular, some sufficient requirements are obtained in order the mentioned general binary operations are t-norms or t-conorms.

The article is organized as follows: Section 2 recalls some definitions and properties about FSs, IVFSs, and T2FSs, provides background related to t-norms and t-conorms on FSs, IVFSs and T2FSs, and presents the "basic" and "restrictive" axioms. In Section 3 the operations $\boldsymbol{\Delta}$ and $\boldsymbol{\nabla}$ (see Definition 14) are proposed. A deeply study is made in order to obtain the necessary properties they have to satisfy to be t-norms or $\mathbf{t}$-conorms both on $\mathbf{L}$ and on M.

Last Section is devoted to expose some conclusions.

\section{Preliminaries}

In this Section, we will recall some concepts and results, in order to understand without difficulty the rest of the paper. Through all the paper, let $X \neq \emptyset$ represents the universe of discourse. Besides, the standard order relation on the real numbers will be denoted by $\leq$.

\subsection{Some fuzzy sets extensions}

Definition 1. ( $[20]$ ) A fuzzy set (FS), $A$, is characterized by a membership function $\mu_{A}$,

$$
\mu_{A}: X \rightarrow[0,1]
$$

where $\mu_{A}(x)$ is the degree of membership of an element $x \in X$ in the set $A$.

Definition 2. ( $[1,2,17])$ An interval-valued fuzzy set (IVFS), $A$, is characterized by a membership function $\sigma_{A}$,

$$
\sigma_{A}: X \rightarrow I=\{[a, b] ; 0 \leq a \leq b \leq 1\} .
$$

So, the degree of membership of an element $x \in X$ is an interval in $[0,1]$. 
Definition 3. ( $[12,13])$ A type-2 fuzzy set (T2FS), $A$, is characterized by a membership function:

$$
\mu_{A}: X \rightarrow \mathbf{M}=[0,1]^{[0,1]}=\operatorname{Map}([0,1],[0,1]) .
$$

That is, $\mu_{A}(x)$ is a fuzzy set in the interval $[0,1]$, and is the degree of membership of an element $x \in X$ in the set $A$. Then

$$
\mu_{A}(x)=f_{x},
$$

where

$$
f_{x}:[0,1] \rightarrow[0,1] .
$$

The set of all type- 2 fuzzy sets on $X$ is denoted by $F_{2}(X)$.

Definition 4. ([18]) Let $a \in[0,1]$. The characteristic function of $a$ is $\mathbf{a}:[0,1] \rightarrow\{0,1\}$, where

$$
\mathbf{a}(x)= \begin{cases}1 & \text { if } x=a \\ 0 & \text { if } x \neq a\end{cases}
$$

Let $\mathbf{J} \subset \mathbf{M}$ be the set of all characteristic functions of elements in $[0,1]$. That is, $\mathbf{J}=\{\mathbf{a}:[0,1] \rightarrow$ $\{0,1\} ; a \in[0,1]\}$. Note that we could establish an equivalence between $\mathbf{J}$ and the values of membership of any fuzzy set.

Definition 5. ([18]) Let $[a, b] \subset[0,1]$. The characteristic function of $[a, b]$ is $[\mathbf{a}, \mathbf{b}]:[0,1] \rightarrow\{0,1\}$, where

$$
[\mathbf{a}, \mathbf{b}](x)= \begin{cases}1 & \text { if } x \in[a, b] \\ 0 & \text { if } x \notin[a, b]\end{cases}
$$

Let $\mathbf{K} \subset \mathbf{M}$ be the set of all characteristic functions of subintervals of $[0,1]$. Also, there exists an equivalence between $\mathbf{K}$ and the values of membership of any interval-valued fuzzy set.

Additionally, as justified in [18], operations in $\operatorname{Map}(X, \mathbf{M})$ can be defined naturally from operations in $\mathbf{M}$ and satisfy the same properties. In this paper, therefore, we will work on $\mathbf{M}$ as all the results can be extended directly and easily to $\operatorname{Map}(X, \mathbf{M})$, which is the set of membership functions of elements in $F_{2}(X)$.

Definition 6. A decreasing function $n:[0,1] \rightarrow$ $[0,1]$ such that $n(0)=1$ and $n(1)=0$, is said to be a negation. If, additionally, $n(n(x))=x$ holds for all $x \in[0,1]$, it is said to be a strong negation.

Definition 7. ([8, 18]) The $\sqcup$ (union), $\sqcap$ (intersection) and $\neg$ operations and the elements $\overline{0}$ and $\overline{1}$ are defined in $\mathbf{M}$ as follows:

$$
\begin{gathered}
(f \sqcup g)(x)=\sup \{f(y) \wedge g(z): y \vee z=x\}, \\
(f \sqcap g)(x)=\sup \{f(y) \wedge g(z): y \wedge z=x\}, \\
\neg f(x)=\sup \{f(y): 1-y=x\}=f(1-x), \\
\overline{0}(x)=\left\{\begin{array}{ll}
1 & \text { if } x=0 \\
0 & \text { if } x \neq 0
\end{array}, \quad \overline{1}(x)=\left\{\begin{array}{ll}
1 & \text { if } x=1 \\
0 & \text { if } x \neq 1 .
\end{array},\right.\right.
\end{gathered}
$$

where $\vee$ and $\wedge$ are maximum and minimum operations, respectively, in the lattice $[0,1]$. Note that $\overline{0}$ and $\overline{1}$ are just the characteristic functions of 0 and 1 , respectively.

It is easy to prove that $\sqcup$ and $\sqcap$ satisfy De Morgan's laws with respect to given operation $\neg$, but $\mathbb{M}=(\mathbf{M}, \sqcup, \sqcap, \neg, \overline{0}, \overline{1})$ does not have a lattice structure, as the absorption law does not hold [8, 18]. Furthermore, the operations $\sqcup$ and $\sqcap$ satisfy the properties required for each one to define a partial order on $\mathbf{M}$.

Definition 8. ([13, 18]) The following two partial orders are defined on $\mathbf{M}$ :

$$
\begin{aligned}
& f \sqsubseteq g \text { if } f \sqcap g=f ; \\
& f \preceq g \text { if } f \sqcup g=g .
\end{aligned}
$$

Generally, these two orders are not the same $[13,18] . \overline{1}$ is the greatest element of the partial order $\sqsubseteq$, because $f \sqsubseteq \overline{1}, \forall f \in \mathbf{M}$; and $\overline{0}$ is the least element of the partial order $\preceq$, as $\overline{0} \preceq f$, $\forall f \in \mathbf{M}([18])$. Moreover, the constant function $g=0(g(x)=0, \forall x \in[0,1])$ is the least and greatest element of $\sqsubseteq$ and $\preceq$, respectively.

In order to facilitate the operations in $\mathbf{M}$, the following Definition and Theorems were given in previous works.

Definition 9. ( $[8,18]$ ) If $f \in \mathbf{M}$, the functions $f^{L}, f^{R} \in \mathbf{M}$ are defined as

$$
\begin{aligned}
& f^{L}(x)=\sup \{f(y): y \leq x\}, \\
& f^{R}(x)=\sup \{f(y): y \geq x\} .
\end{aligned}
$$

Now the following result can be established.

Theorem 1. ([8, 18]) Let $f, g \in \mathbf{M}$. The equalities:

$$
\begin{aligned}
& f \sqcup g=\left(f \wedge g^{L}\right) \vee\left(f^{L} \wedge g\right)=(f \vee g) \wedge\left(f^{L} \wedge g^{L}\right), \\
& f \sqcap g=\left(f \wedge g^{R}\right) \vee\left(f^{R} \wedge g\right)=(f \vee g) \wedge\left(f^{R} \wedge g^{R}\right) .
\end{aligned}
$$

hold.

And we have a characterization for each of the partial orders $\sqsubseteq$ and $\preceq$.

Theorem 2. ([18]) Let $f, g \in \mathbf{M}$. Then :

$$
\begin{gathered}
f \sqsubseteq g \Leftrightarrow\left(f^{R} \wedge g\right) \leq f \leq g^{R}, \\
f \preceq g \Leftrightarrow\left(g^{L} \wedge f\right) \leq g \leq f^{L} .
\end{gathered}
$$

Additionally, note that the characteristic function of an interval $[a, b]$ is just $[\mathbf{a}, \mathbf{b}]=\mathbf{a}^{L} \wedge \mathbf{b}^{R}$ (see [18]).

Next, we are going to consider a special kind of functions in $\mathbf{M}$. This will allow us to obtain a bounded and complete lattice, and then construct t-norms and t-conorms properly. Let us recall that:

Definition 10. Let $f \in \mathbf{M}$. It is said that $f$ is normal if $\sup \{f(x): x \in[0,1]\}=1$. 
Let $\mathbf{N}$ be the set of all normal functions in $\mathbf{M}$. Note that if $f \in \mathbf{M}$, then $f \in \mathbf{N}$ if and only if $f^{L} \vee f^{R}=1$.

Definition 11. Let $f \in \mathbf{M}$. It is said that $f$ is convex, if for any $x \leq y \leq z$, the inequality $f(y) \geq$ $f(x) \wedge f(z)$ holds.

Let $\mathbf{C}$ be the set of all convex functions on $\mathbf{M}$. If $f \in \mathbf{M}$, then $f \in \mathbf{C}$ if and only if $f=f^{L} \wedge f^{R}$.

The set of all normal and convex functions of $f \in \mathbf{M}$ will be denoted $\mathbf{L}$. The algebra $\mathbb{L}=$ $(\mathbf{L}, \sqcup, \sqcap, \neg, \overline{0}, \overline{1})$ is a subalgebra of $\mathbb{M}$. In $\mathbf{L}$, the partial orders $\sqsubseteq$ and $\preceq$ are equivalent, and $\mathbb{L}$ is a bounded ( $\overline{0}$ and $\overline{1}$ are the minimum and the maximum, respectively) and complete lattice (see $[7,8,13,18])$. Furthermore, that $\mathbf{J} \subset \mathbf{K} \subset \mathbf{L} \subset \mathbf{M}$ is obvious.

The following characterization will help to establish new results.

Theorem 3. ( $[7,8])$ Let $f, g \in \mathbf{L} . \quad f \sqsubseteq g$ if and only if

$$
g^{L} \leq f^{L} \quad \text { y } f^{R} \leq g^{R} .
$$

\subsection{T-norms and t-conorms}

Up to now, we have only considered the operations introduced in Definition 7. But from the Zadeh's Extension Principle $[12,13,21]$ some new operations can be obtained using not only the minimum, maximum, and standard negation, but also other operations. In this direction, we introduced negations in partially ordered sets, and we gave some negations in $\mathbf{M}$.

Definition 12. Let $\mathbf{M}$ be the set of all fuzzy sets on $[0,1]$ and $n$ a suprajective negation in $[0,1]$. The operation $N_{n}: \mathbf{M} \rightarrow \mathbf{M}$ is given, for any $f \in \mathbf{M}$ by:

$$
\left(N_{n}(f)\right)(x)=\sup \{f(y): n(y)=x\} \forall x \in[0,1] .
$$

We proved that $N_{n}$ is a negation on $\mathbf{L}$ (that is, decreasing in $(\mathbf{L}, \sqsubseteq)$ with $N_{n}(\overline{0})=\overline{1}$ and $N_{n}(\overline{1})=\overline{0}$ ), that is strong (involutive) if and only if $n$ is strong.

On the other hand, recall that a t-norm $([9])$ is a binary operation $T:[0,1]^{2} \rightarrow[0,1]$, commutative, associative, increasing on each argument, and with neutral element 1. More, a t-conorm is a binary operation $S:[0,1]^{2} \rightarrow[0,1]$, commutative, associative, increasing on each argument, and with neutral element 0. Similar definitions apply to bounded lattices. In $[5,19]$ this definition was extended both to IVFSs and to T2FSs, adding some axioms in order to collect some desirable properties. For example, as $\mathbf{J} \subset \mathbf{K} \subset \mathbf{L}$, it seems reasonable to demand t-norms on $\mathbf{L}$ to be closed both on $\mathbf{J}$ and on $\mathbf{K}$. Furthermore, as t-norms on IVFSs satisfy $T([1,1],[a, b])=[a, b]$ and $T([0,0],[a, b])=[0,0]$, by analogy the condition $T([0,1],[a, b])=[0, b]$ is required. So the following "restrictive" axioms were established:
Definition 13. ([19]) The binary operation $T$ : $\mathbf{L}^{2} \rightarrow \mathbf{L}$ is a t-norm on $\mathbf{L}$ if:

1. $T$ is commutative

2. $T$ is associative.

3. $T(f, \overline{1})=f$ for any $f \in \mathbf{L}$ (neutral element).

4. If $g \sqsubseteq h$ then $T(f, g) \sqsubseteq T(f, h)$, for all $f, g, h \in$ $\mathbf{L}$ (increasing on each argument).

5. $T\left(\left(\mathbf{0}^{L} \wedge \mathbf{1}^{R}\right),\left(\mathbf{a}^{L} \wedge \mathbf{b}^{R}\right)\right)=\left(\mathbf{0}^{L} \wedge \mathbf{b}^{R}\right)$.

6. $T$ is closed in $\mathbf{J}$.

7. $T$ is closed in $\mathbf{K}$.

Similarly, a binary operation $S: \mathbf{L}^{2} \rightarrow \mathbf{L}$ is a t-conorm if it satisfies all the axioms of t-norm, but the axiom 3 (as in this case the neutral element should be $\overline{0}$ ), and the axiom 5 (that now will be $\left.S\left(\left(\mathbf{0}^{L} \wedge \mathbf{1}^{R}\right),\left(\mathbf{a}^{L} \wedge \mathbf{b}^{R}\right)\right)=\left(\mathbf{a}^{L} \wedge \mathbf{1}^{R}\right)\right)$. Axioms 1, 2,3 and 4 , will be called "basic" axioms.

\section{T-norms and t-conorms on $\mathrm{L}$}

In $[17,18,19]$ it was proved that the operations $\sqcap$ and $\sqcup$ satisfy the "restrictive" axioms of t-norm and t-conorm on $\mathbf{L}$, respectively, given in Definition 13 . More, two new families of operations, also satisfying "restrictive" axioms on $\mathbf{L}$, were introduced on $\mathbf{M}$ :

$$
\begin{gathered}
(f \overline{\mathbf{\Delta}} g)(x)=\sup \{f(y) \wedge g(z): y \Delta z=x\}, \\
(f \bar{\nabla} g)(x)=\sup \{f(y) \wedge g(z): y \nabla z=x\},
\end{gathered}
$$

where $\Delta$ and $\nabla$ are continuous t-norm and t-conorm, respectively, on $[0,1]$.

In the following our main goal is to obtain a broader set of operations on $\mathbf{M}$, and study the necessary requirements to in fact to be t-norms in the restrictive sense. In this direction, let us begin with the following definition.

Definition 14. Let $\star$ be a binary operation on $[0,1], \Delta$ a t-norm and $\nabla$ a t-conorm on $[0,1]$. The binary operations $\boldsymbol{\Lambda}$ and $\boldsymbol{\nabla}$ on $\mathbf{M}$ are given for any $f, g \in \mathbf{M}$ as:

$$
\begin{aligned}
& (f \mathbf{\Delta} g)(x)=\sup \{f(y) \star g(z): y \Delta z=x\}, \\
& (f \nabla g)(x)=\sup \{f(y) \star g(z): y \nabla z=x\} .
\end{aligned}
$$

Note that $\boldsymbol{\Delta}=\overline{\boldsymbol{\Lambda}}$ and $\boldsymbol{\nabla}=\overline{\boldsymbol{\nabla}}$, just in case $\star=\wedge$.

A first result is:

Theorem 4. (De Morgan's Laws) Let $n$ be a strong negation on $[0,1]$. If $\Delta$ and $\nabla$, are $n$-dual (for all $x, y \in[0,1], n(x \triangle y)=n(x) \nabla n(y))$, then

$$
\begin{gathered}
N_{n}(f \mathbf{\Delta} g)=N_{n}(f) \mathbf{\nabla} N_{n}(g), \\
N_{n}(f \mathbf{\nabla} g)=N_{n}(f) \mathbf{\Delta} N_{n}(g), \forall f, g \in \mathbf{M} .
\end{gathered}
$$

That is, $\boldsymbol{\Delta}$ are $\boldsymbol{\nabla}$ dual respect to $N_{n}$, provided $\triangle$ and $\nabla$ are dual respect to $n$.

The proofs of the two following propositions are straightforward. 
Proposition 1. Let $\boldsymbol{\Delta}$ and $\boldsymbol{\nabla}$ as in Definition 14. $\boldsymbol{\Delta}$ and $\boldsymbol{\nabla}$ are commutative if and only if $\star$ is commutative.

Proposition 2. If the operation $\star$ in Definition 14 is continuous and increasing on each variable, then $\boldsymbol{\Delta}$ and $\boldsymbol{\nabla}$ are associative if and only if $\star$ is associative.

Proposition 3. Let $c \in[0,1]$, and the function $c:[0,1] \rightarrow[0,1]$ given $\forall x \in[0,1]$ as $c(x)=c$. Then for all $f \in \mathbf{M}$ it is

$$
\begin{aligned}
& (f \mathbf{\Delta} c)(x)=\sup \{f(y) \star c: x \leq y\}, \\
& (f \boldsymbol{\nabla} c)(x)=\sup \{f(y) \star c: x \geq y\} .
\end{aligned}
$$

provided $\Delta$ and $\nabla$ are continuous in Definition 14 .

Proof. $(f \boldsymbol{\Delta} c)(x)=\sup \{f(y) \star c(z): y \Delta z=x\}=$ $\sup \{f(y) \star c: y \Delta z=x\}$. As $\Delta$ is a t-norm (and then $\Delta \leq$ Min) , $x=y \Delta z \leq y \wedge z$ and $x \leq y$. More, if $x \leq y$ then , by continuity, it exists a $z \in[0,1]$ such that $x=y \Delta z$. Then, $(f \mathbf{\Delta} c)(x)=\sup \{f(y) \star$ $c: x \leq y\}, \forall x \in[0,1]$.

The proof of the second equality is similar.

Corollary 1. In the same conditions as in Proposition 3 ,

1. If $c$ is a neutral element of the operation $\star$, then $f \mathbf{\Delta} c=f^{R}$, and $f \mathbf{\nabla} c=f^{L}$.

2. If $\star$ is a t-norm on $[0,1]$, then $f \boldsymbol{\Delta} 1=f^{R}$, and $f \boldsymbol{\nabla} 1=f^{L}$, where $1 \in \mathbf{M}$ is the function given by $1(x)=1 \forall x \in[0,1]$.

3. If $\star$ is a t-conorm on $[0,1]$, then $f \mathbf{\Delta} 0=f^{R}$, and $f \boldsymbol{\nabla} 0=f^{L}$, where $0(x)=0 \forall x \in[0,1]$.

4. If $c$ is an absorbent element of the operation $\star$, then $f \mathbf{\Delta} c=c$, and $f \mathbf{\nabla} c=c$.

5. If $\star$ is a t-norm on $[0,1]$, then $f \mathbf{\Delta} 0=f \boldsymbol{\nabla} 0=0$.

6. If $\star$ is a t-conorm on $[0,1]$, then $f \boldsymbol{\Delta} 1=f \mathbf{\nabla} 1=$ 1 .

Remark 1. In [18], section 5, proposition 61, the authors maintain that if $\star=\wedge$ and $\Delta$ any t-norm, then $f \mathbf{\Delta} 1=f^{R}$ for any $f \in \mathbf{M}$. Nevertheless this assertion is not correct, as the continuity of $\Delta$ should be demanded. In fact, let us consider, for example, $\star=\wedge$ and the non continuous t-norm

$$
x \Delta y=\left\{\begin{array}{lc}
x & \text { if } y=1 \\
y & \text { if } x=1 \\
0 & \text { otherwise }
\end{array}\right.
$$

and the function

$$
f(x)=\left\{\begin{array}{cc}
0.3 & \text { if } x \neq 0.5 \\
1 & \text { if } x=0.5
\end{array}\right.
$$

In this case, $(f \mathbf{\Delta} 1)(0.1)=(f(0.1) \wedge 1(1)) \vee(f(1) \wedge$ $1(0.1))=(0.3 \wedge 1) \vee(0.3 \wedge 1)=0.3 \neq 1=f^{R}(0.1)$.

Corollary 2. Let $\boldsymbol{\Delta}$ and $\boldsymbol{\nabla}$ be the operations given in Definition 14. $\forall f \in \mathbf{M}$ we have:
1. If $u \star 1=u$ and $u \star 0=0 \forall u \in[0,1]$, then

$$
\begin{aligned}
& f \mathbf{\Delta} \overline{1}=f, \\
& (f \mathbf{\Delta} \overline{0})(x)=\left\{\begin{array}{cc}
\sup f & \text { if } x=0 \\
0 & \text { if } x \neq 0
\end{array},\right.
\end{aligned}
$$

Additionally, if $f \in \mathbf{N}$ then $f \mathbf{\Delta} \overline{0}=\overline{0}$.

2. If $u \star 1=1 \forall u \in[0,1]$, then $f \mathbf{\Delta} \overline{1}=1$.

3. If $u \star 1=1$ and $u \star 0=u \forall u \in[0,1]$, and $\triangle$ is continuous, then $f \mathbf{\Delta} \overline{0}=\overline{0} \vee f^{R}$.

4. If $u \star 1=u$ and $u \star 0=0 \forall u \in[0,1]$, then $f \boldsymbol{\nabla} \overline{0}=f$,

$$
(f \boldsymbol{\nabla} \overline{1})(x)=\left\{\begin{array}{cc}
\sup f & \text { if } x=1 \\
0 & \text { if } x \neq 1
\end{array},\right.
$$

Additionally, if $f \in \mathbf{N}$ then $f \mathbf{\nabla} \overline{1}=\overline{1}$.

5. If $u \star 1=1 \forall u \in[0,1]$, then $f \boldsymbol{\nabla} \overline{0}=1$.

6. If $u \star 1=1$ and $u \star 0=u \forall u \in[0,1]$, and $\nabla$ is continuous, then $f \mathbf{\nabla} \overline{1}=\overline{1} \vee f^{L}$.

Proposition 4. Let $\triangle, \nabla$ and $\star$ the operations in Definition 14. If $\Delta$ and $\nabla$ are continuous, and $\star$ is commutative, associative, continuous, increasing in each argument and with a neutral element $c$, then $\forall f, g \in \mathbf{M}$,

$$
\begin{aligned}
& (f \mathbf{\Delta} g)^{R}=f \mathbf{\Delta} g^{R}=f^{R} \mathbf{\Delta} g=f^{R} \mathbf{\Delta} g^{R}, \\
& (f \mathbf{\nabla} g)^{L}=f \boldsymbol{\nabla} g^{L}=f^{L} \mathbf{\nabla} g=f^{L} \mathbf{\nabla} g^{L} .
\end{aligned}
$$

Proof. Let $c$ be the function given by $c(x)=c, \forall x \in[0,1]$.

From Corollary 1, $(f \mathbf{\Delta} g) \mathbf{\Delta} c=(f \mathbf{\Delta} g)^{R}$ holds.

By Proposition 2, $(f \mathbf{\Delta} g) \mathbf{\Delta} c=f \mathbf{\Delta}(g \mathbf{\Delta} c)=f \mathbf{\Delta} g^{R}$.

And, as operation $\star$ is commutative,

$(f \mathbf{\Delta} g) \mathbf{\Delta} c=(f \mathbf{\Delta} c) \mathbf{\Delta} g=f^{R} \mathbf{\Delta} g$.

Then, $(f \boldsymbol{\Delta} g)^{R}=f \boldsymbol{\Delta} g^{R}=f^{R} \boldsymbol{\Delta} g$, and

$(f \mathbf{\Delta} g)^{R}=\left((f \mathbf{\Delta} g)^{R}\right)^{R}=\left(f \mathbf{\Delta} g^{R}\right)^{R}=f^{R} \mathbf{\Delta} g^{R}$.

The rest of the equalities have similar proofs.

Due to limitation of the length of this work, the following results will be stated without proof.

Proposition 5. If $\star$ is increasing on each argument, $\forall f, g \in \mathbf{M}(f \mathbf{\nabla} g)^{R}=f^{R} \mathbf{\nabla} g^{R} \Leftrightarrow \star$ is continuous.

Proposition 6. If $\star$ continuous and increasing on each argument, then $\forall f, g \in \mathbf{M}$

$$
(f \mathbf{\Delta} g)^{L}=f^{L} \boldsymbol{\Delta} g^{L} .
$$

Proposition 7. If $\star$ is continuous, increasing on each argument and satisfies $1 \star 1=1$, then both $\boldsymbol{\Delta}$ and $\boldsymbol{\nabla}$ are closed on $\mathbf{N}$.

Proposition 8. If $\star$ is increasing on each variable, the following stamens

$$
\begin{aligned}
& f \mathbf{\Delta}(g \vee h)=(f \mathbf{\Delta} g) \vee(f \mathbf{\Delta} h), \\
& f \mathbf{\nabla}(g \vee h)=(f \boldsymbol{\nabla} g) \vee(f \boldsymbol{\nabla} h), \\
& f \mathbf{\Delta}(g \wedge h) \leq(f \boldsymbol{\Delta} g) \wedge(f \mathbf{\Delta} h), \\
& f \mathbf{\nabla}(g \wedge h) \leq(f \mathbf{\nabla} g) \wedge(f \mathbf{\nabla} h),
\end{aligned}
$$

hold $\forall f, g, h \in \mathbf{M}$, where $\leq$ is the usual order in the set of functions ( $f \leq g$ if and only if $f(x) \leq$ $g(x), \forall x)$. 
Corollary 3. If $\star$ is increasing on each argument, then $\forall f, g, h \in \mathbf{M}$, such that $g \leq h$, the inequalities

$$
(f \mathbf{\Delta} g) \leq(f \mathbf{\wedge} h),(f \mathbf{\nabla} g) \leq(f \mathbf{\nabla} h),
$$

hold.

Proposition 9. If $\star$ is continuous and increasing on each argument, then , $\forall f, g, h \in \mathbf{M}$

$$
\begin{aligned}
& f \mathbf{\Delta}(g \sqcup h) \leq(f \mathbf{\Delta} g) \sqcup(f \mathbf{\Delta} h), \\
& f \mathbf{\nabla}(g \sqcap h) \leq(f \mathbf{\nabla} g) \sqcap(f \boldsymbol{\nabla} h) .
\end{aligned}
$$

Proposition 10. Let consider $\Delta$ and $\nabla$ continuous. And let $\star$ be an operation commutative, associative, continuous, with neutral element and increasing on each argument. $\forall f, g, h \in \mathbf{M}$, the inequalities

$$
\begin{aligned}
& f \mathbf{\Delta}(g \sqcap h) \leq(f \mathbf{\Delta} g) \sqcap(f \mathbf{\Delta} h), \\
& f \mathbf{\nabla}(g \sqcup h) \leq(f \mathbf{\nabla} g) \sqcup(f \mathbf{\nabla} h),
\end{aligned}
$$

hold.

Although Propositions 9 and 10 only establish inequalities, the following results will provide some sufficient conditions in order to $\boldsymbol{\Delta}$ and $\mathbf{\nabla}$ satisfy the distributivity laws respect to $\sqcap$ and $\sqcup$, and the increasing monotonicity.

Proposition 11. Let $\Delta$ and $\nabla$ continuous. And $\star$ commutative, associative, continuous, with neutral element and increasing on each argument. Given $f, g, h \in \mathbf{M}$,

- if $g \sqsubseteq h$, and $g^{R} \leq h$ or $g^{R} \geq h$, then

$$
(f \mathbf{\Delta} g)=(g \mathbf{\Delta} f) \sqsubseteq(f \mathbf{\Delta} h)=(h \mathbf{\Delta} f) .
$$

- if $g \preceq h$, and $h^{L} \leq g$ or $h^{L} \geq g$, then

$$
(f \mathbf{\nabla} g)=(g \nabla f) \preceq(f \mathbf{\nabla} h)=(h \mathbf{\nabla} f) .
$$

Proposition 12. Let $\Delta$ and $\nabla$ continuous. And let $\star$ continuous, increasing on each argument, such that $a \star(b \wedge c) \geq(d \star b) \wedge(e \star c)$, provided that $a \geq(d \wedge e)$. For all $f \in \mathbf{C}$ and $g, h \in \mathbf{M}$, the equalities

$$
\begin{aligned}
& f \mathbf{\Delta}(g \sqcup h)=(f \mathbf{\Delta} g) \sqcup(f \mathbf{\Delta} h), \\
& f \mathbf{\nabla}(g \sqcap h)=(f \mathbf{\nabla} g) \sqcap(f \boldsymbol{\nabla} h) .
\end{aligned}
$$

hold. Additionally, if $\star$ is commutative, associative and with neutral element, then we have that

$$
\begin{aligned}
& f \mathbf{\Delta}(g \sqcap h)=(f \mathbf{\Delta} g) \sqcap(f \mathbf{\Delta} h), \\
& f \mathbf{\nabla}(g \sqcup h)=(f \mathbf{\nabla} g) \sqcup(f \boldsymbol{\nabla} h) .
\end{aligned}
$$

Corollary 4. Let $\Delta$ and $\nabla$ continuous. And let $\star$ continuous, commutative, increasing on each argument, and such that $a \star(b \wedge c) \geq(d \star b) \wedge(e \star c)$, provided that $a \geq(d \wedge e)$. For all $f \in \mathbf{C}$ and $g, h \in \mathbf{M}$,

- if $g \preceq h$, then

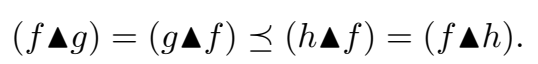

- if $g \sqsubseteq h$, then

$$
(f \mathbf{\nabla} g)=(g \mathbf{\nabla} f) \sqsubseteq(h \mathbf{\nabla} f)=(f \mathbf{\nabla} h) .
$$

Additionally, if $\star$ is associative and with neutral element,

- if $g \sqsubseteq h$, then

$$
(f \mathbf{\Delta} g)=(g \mathbf{\Delta} f) \sqsubseteq(h \mathbf{\Delta} f)=(f \mathbf{\Delta} h),
$$

- if $g \preceq h$, then

$$
(f \mathbf{\nabla} g)=(g \mathbf{\nabla} f) \preceq(h \mathbf{\nabla} f)=(f \mathbf{\nabla} h) .
$$

Remark 2. - The Minimum t-norm $\wedge$ fulfills all conditions of Proposition 12, and consequently, if both $\Delta$ and $\nabla$ are continuous, and $f$ is convex, we can assert that $\boldsymbol{\Lambda}$ and $\boldsymbol{\nabla}$, determined by $\star=\wedge$, are distributive respect to $\Pi$ and $\sqcup$, as showed in [18].

- The only t-norm on $[0,1]$ satisfying the condition $a \star(b \wedge c) \geq(d \star b) \wedge(e \star c)$, provided that $a \geq(d \wedge e)$, is the Minimun. Because if $\star$ is not the Minimun, there exist $a, b \in[0,1]$, such that $a \star b<\operatorname{Min}(a, b)$ (recall the Min is the greatest t-norm). For these values we have

$$
a \star(1 \wedge b)=a \star b<a \wedge b=(a \star 1) \wedge(1 \star b),
$$

and, however, $a>(a \wedge 1)$.

- If $\alpha$ is an automorphism on $[0,1]$, the operation $\star$ given by $x \star y=\alpha(x) \wedge \alpha(y)$, for all $x, y \in[0,1]$, is continuous, commutative and increasing on each variable, and $\alpha(a) \wedge \alpha(b \wedge c) \geq(\alpha(d) \wedge$ $\alpha(b)) \wedge(\alpha(e) \wedge \alpha(c))$, provided that $a \geq(d \wedge e)$, but it is not associative.

- The operation $x \star y=x \vee y$ (Maximum tconorm) does not fulfill the condition $a \star(b \wedge$ $c) \geq(d \star b) \wedge(e \star c)$, provided that $a \geq(d \wedge e)$. For example, $\quad(0.5 \vee(0.1 \wedge 0.8)=0.5 \ngtr$ $(1 \vee 0.1) \wedge(0.4 \vee 0.8)=0.8$

Corollary 5. Let $\boldsymbol{\Lambda}$ and $\boldsymbol{\nabla}$ be the operations determined by $\Delta$ and $\nabla$ continuous, and by $\star(x, y)=$ $\alpha(x) \wedge \alpha(y)$, for all $x, y \in[0,1]$, where $\alpha$ is an automorphism on $[0,1]$. For all $f \in \mathbf{C}$ and $g, h \in \mathbf{M}$, the assertions

$$
\begin{gathered}
f \mathbf{\Delta}(g \sqcup h)=(f \mathbf{\Delta} g) \sqcup(f \mathbf{\Delta} h), \\
f \mathbf{\nabla}(g \sqcap h)=(f \mathbf{\nabla} g) \sqcap(f \mathbf{\nabla} h), \\
\text { if } g \preceq h \Rightarrow(f \mathbf{\Delta} g)=(g \mathbf{\Delta} f) \preceq(h \mathbf{\Delta} f)=(f \mathbf{\Delta} h), \\
\text { if } g \sqsubseteq h \Rightarrow(f \mathbf{\nabla} g)=(g \mathbf{\nabla}) \sqsubseteq(h \mathbf{\nabla} f)=(f \mathbf{\nabla} h),
\end{gathered}
$$

hold. 
Proposition 13. Let $\Delta$ and $\nabla$ be continuous operations, and $\star$ an operation continuous, increasing on each argument, such that $u \star 1=u$ and $u \star 0=$ $0, \forall u \in[0,1]$, and satisfying $a \star(b \wedge c) \geq(d \star b) \wedge(e \star c)$, provided that $a \geq(d \wedge e)$. Let $f \in \mathbf{M}$. Then $\forall g, h \in \mathbf{M}$

$$
\begin{aligned}
& f \mathbf{\Delta}(g \sqcup h)=(f \mathbf{\Delta} g) \sqcup(f \mathbf{\Delta} h), \\
& f \boldsymbol{\nabla}(g \sqcap h)=(f \mathbf{\nabla} g) \sqcap(f \boldsymbol{\nabla} h),
\end{aligned}
$$

if and only if $f$ is convex.

Proof. Similar to given in [18], in Theorem 63.

Proposition 14. Let $\Delta$ and $\nabla$, continuous, and * continuous, commutative, increasing on each argument, and satisfying $a \star(b \wedge c) \geq(d \star b) \wedge(e \star c)$, provided $a \geq(d \wedge e)$. Then $\boldsymbol{\Delta}$ and $\boldsymbol{\nabla}$ are closed on C.

Additionally, if $1 \star 1=1$ holds, then $\boldsymbol{\Delta}$ and are closed on $\mathbf{L}$, and increasing respect to the partial order of $\mathbf{L}$.

Now we consider two particular operations:

$$
\begin{aligned}
& (f \ominus g)(x)=\sup \{f(y) \vee g(z): y \wedge z=x\}, \\
& (f \oplus g)(x)=\sup \{f(y) \vee g(z): y \vee z=x\},
\end{aligned}
$$

and we give the following characterizations:

Proposition 15. For all $f, g \in \mathbf{M}$, the equalities

$f \ominus g=\left(f \vee g^{R}\right) \vee\left(f^{R} \vee g\right)=f^{R} \vee g^{R}=(f \vee g)^{R}$,

$f \oplus g=\left(f \vee g^{L}\right) \vee\left(f^{L} \vee g\right)=f^{L} \vee g^{L}=(f \vee g)^{L}$,

hold.

Although the operation $\star=\vee$ do not fulfill the conditions of the Proposition 12, the following results can be proved.

Proposition 16. $\forall f, g, h \in \mathbf{M}$

$$
\begin{aligned}
& f \ominus(g \sqcap h)=(f \ominus g) \sqcap(f \ominus h), \\
& f \oplus(g \sqcup h)=(f \oplus g) \sqcup(f \oplus h) .
\end{aligned}
$$

Corollary 6. The operations $\ominus$ and $\oplus$ are increasing respect to $\sqsubseteq$ and $\preceq$, respectively. That is, given $f, g, h \in \mathbf{M}$,

$$
\begin{aligned}
& \text { if } g \sqsubseteq h \Rightarrow(f \ominus g) \sqsubseteq(f \ominus h) . \\
& \text { if } g \preceq h \Rightarrow(f \oplus g) \preceq(f \oplus h) .
\end{aligned}
$$

Proposition 17. Let $f, g \in \mathbf{M}$, then

$$
(f \ominus g) \in \mathbf{C},(f \oplus g) \in \mathbf{C} .
$$

From Propositions 7 and 17, it is straightforward the following.

Corollary 7. $\ominus$ and $\oplus$ are closed on $\mathbf{L}$.
Furthermore operations $\ominus$ and $\oplus$ are increasing respect to the partial orden on $\mathbf{L}$.

Corollary 8. Consider $f, g, h \in \mathbf{L}$, such that $g \sqsubseteq h$ ( $\sqsubseteq$ is the partial order on $\mathbf{L}$ ). Then

$$
(f \ominus g) \sqsubseteq(f \ominus h),(f \oplus g) \sqsubseteq(f \oplus h) .
$$

Proof. Straightforward from the Corollaries 6 and 7.

In the following, some properties of $\boldsymbol{\Delta}$ and $\boldsymbol{\nabla}$ in the sets $\mathbf{J}$ and $\mathbf{K}$ will be faced.

Proposition 18. Let $\triangle$ and $\nabla$ continuous. And let $\star$ be an operation satisfying $1 \star 0=0 \star 1=0$, $1 \star 1=1$, and $0 \star 0=0$. If $a \leq b$ and $c \leq d$, we have that

$$
\left(\mathbf{a}^{L} \wedge \mathbf{b}^{R}\right) \mathbf{\Delta}\left(\mathbf{c}^{L} \wedge \mathbf{d}^{R}\right)=\mathbf{e}^{L} \wedge \mathbf{f}^{R},
$$

where $e=(a \Delta c) \leq f=(b \Delta d)$. And

$$
\left(\mathbf{a}^{L} \wedge \mathbf{b}^{R}\right) \mathbf{\nabla}\left(\mathbf{c}^{L} \wedge \mathbf{d}^{R}\right)=\mathbf{e}^{L} \wedge \mathbf{f}^{R},
$$

where $e=(a \nabla c) \leq f=(b \nabla d)$.

That is, $\boldsymbol{\Delta}$ and $\boldsymbol{\nabla}$ are closed on $\mathbf{K}$, provided the conditions of the formulation.

Proof. Similar to that given in [18], in Section 5.2.

Corollary 9. In the same conditions as in the previous Proposition 18, we obtain

$$
\begin{aligned}
& \left(\mathbf{0}^{L} \wedge \mathbf{1}^{R}\right) \mathbf{\Delta}\left(\mathbf{a}^{L} \wedge \mathbf{b}^{R}\right)=\mathbf{0}^{L} \wedge \mathbf{b}^{R} . \\
& \left(\mathbf{0}^{L} \wedge \mathbf{1}^{R}\right) \mathbf{\nabla}\left(\mathbf{a}^{L} \wedge \mathbf{b}^{R}\right)=\mathbf{a}^{L} \wedge \mathbf{1}^{R},
\end{aligned}
$$

Corollary 10. In the same conditions as in the Proposition 18, we obtain

$$
(\mathbf{a} \Delta \mathbf{c})=\mathbf{e}
$$

were $e=a \Delta c$. That is, $\boldsymbol{\Delta}$ is closed on $\mathbf{J}$.

$$
(\mathbf{a \nabla c})=\mathbf{m},
$$

were $m=a \nabla c$. That is, $\boldsymbol{\nabla}$ is closed on $\mathbf{J}$.

Proposition 19. Let $\triangle$ and $\nabla$ continuous, and let $\star$ be a t-conorm in $[0,1]$. If $a \leq b$ and $c \leq d$, then

$$
\left(\mathbf{a}^{L} \wedge \mathbf{b}^{R}\right) \mathbf{\Delta}\left(\mathbf{c}^{L} \wedge \mathbf{d}^{R}\right)=\mathbf{0}^{L} \wedge \mathbf{f}^{R},
$$

where $f=b \vee d$.

$$
\left(\mathbf{a}^{L} \wedge \mathbf{b}^{R}\right) \mathbf{\nabla}\left(\mathbf{c}^{L} \wedge \mathbf{d}^{R}\right)=\mathbf{f}^{L} \wedge \mathbf{1}^{R},
$$

where $f=a \wedge c$. That is, $\boldsymbol{\Delta}$ and $\boldsymbol{\nabla}$ are closed on $\mathbf{K}$ provided $\Delta$ and $\nabla$ are continuous, and $\star$ is a t-conorm. 
Corollary 11. In the same conditions as in the previous Proposition 19, we have that

$$
\begin{gathered}
\left(\mathbf{0}^{L} \wedge \mathbf{1}^{R}\right) \mathbf{\Delta}\left(\mathbf{a}^{L} \wedge \mathbf{b}^{R}\right)=\mathbf{0}^{L} \wedge \mathbf{1}^{R} . \\
(\mathbf{a} \mathbf{\Delta} \mathbf{c})=\mathbf{0}^{L} \wedge \mathbf{f}^{R},
\end{gathered}
$$

where $f=a \vee c$. That is, $\boldsymbol{\Delta}$ is not closed on $\mathbf{J}$.

$$
\begin{gathered}
\left(\mathbf{0}^{L} \wedge \mathbf{1}^{R}\right) \mathbf{\nabla}\left(\mathbf{a}^{L} \wedge \mathbf{b}^{R}\right)=\mathbf{0}^{L} \wedge \mathbf{1}^{R} . \\
(\mathbf{a} \mathbf{c} \mathbf{c})=\mathbf{f}^{L} \wedge \mathbf{1}^{R},
\end{gathered}
$$

where $f=a \wedge c$. That is, $\nabla$ is not closed on $\mathbf{J}$.

Summarizing this Section 3 , if $\star$ is not commutative, $\boldsymbol{\Delta}$ and $\boldsymbol{\nabla}$ are not commutative, therefore, they are not t-norm neither t-conorm, respectively, on both $\mathbf{L}$ and $\mathbf{M}$. More, if $\star$ is a t-conorm in $[0,1]$, then $\boldsymbol{\Delta}$ and $\boldsymbol{\nabla}$ do not satisfy axioms 3, 5 and 6 of the definition 13 .

If $\Delta$ and $\nabla$ are continuous, and $x \star y=\alpha(x) \wedge \alpha(y)$, for all $x, y \in[0,1]$, where $\alpha$ is an automorphism on $[0,1]$, then $\boldsymbol{\Delta}$ and $\boldsymbol{\nabla}$ satisfy all "restrictive" axioms of t-norm and t-conorm on $\mathbf{L}$, respectively, except the associativity and the neutral element. The problem of obtaining a binary operation $\star$, apart from the Minimum, in order to $\boldsymbol{\Delta}$ and $\boldsymbol{\nabla}$ be t-norm and tconorm, respectively, on $\mathbf{L}$, has not been solved yet.

Moreover, if $\Delta$ is continuous, and $\star$ is a continuous t-norm, then $\boldsymbol{\Delta}$ is commutative, associative, $\overline{1}$ is the neutral element, and, given $f, g, h \in \mathbf{M}$, - if $g \sqsubseteq h$, and $g^{R} \leq h$ or $g^{R} \geq h$, then

$$
(f \mathbf{\Delta} g) \sqsubseteq(f \mathbf{\Delta} h) .
$$

Namely, in these conditions, $\boldsymbol{\Delta}$ fulfills all "basic" axioms of t-norm on (M, $\mathbf{M})$. Similarly, if $\nabla$ is continuous, and $\star$ is a continuous t-norm, then $\boldsymbol{\nabla}$ is commutative, associative, $\overline{0}$ is the neutral element, and, given $f, g, h \in \mathbf{M}$,

- if $g \preceq h$, and $h^{L} \leq g$ or $h^{L} \geq g$, then

$$
(f \boldsymbol{\nabla} g) \preceq(f \nabla h) .
$$

That is, in these conditions, $\nabla$ fulfills all "basic" axioms of t-conorm on $(\mathbf{M}, \preceq)$.

\section{Conclusions}

In this study the operations $\boldsymbol{\Delta}$ and $\boldsymbol{\nabla}$ have been defined on $\mathbf{M}$. They are more general than those given in [18]. Among other properties, it has been studied in which conditions each of the "restrictive" axioms of t-norm and t-conorm, is satisfied. This deeply analysis has been made on $\mathbf{L}$ as well as on $\mathbf{M}$. From this study it has been determined, for example, that if $\star$ is not commutative, or if it is a t-conorm, then $\boldsymbol{\Delta}$ and $\boldsymbol{\nabla}$ are not t-norm and t-conorm, respectively. However, new t-norms and t-conorms have been deducted according to the "basic" axioms. Further, new operations are determined that satisfy the distributive laws respect to $\sqcap$ and $\sqcup$.

An open problem is to determine different operations to $\overline{\boldsymbol{\Lambda}}$ and $\overline{\boldsymbol{\nabla}}$ be t-norm and t-conorm, respectively, in the "restrictive" sense, on $\mathbf{L}$.

\section{Acknowledgements}

This paper was partially supported by CICYT (Spain) project TIN2011-29827-C02-01, UPM-CAM, FONACIT (Venezuela) and UNET (Venezuela).

\section{References}

[1] H. Bustince, E. Barrenechea and M. Pagola, Generation of interval-valued fuzzy and Atanassov's intuitionistic fuzzy connectives from fuzzy connectives and from $K_{\alpha}$ operators. Laws for conjunctions and disjunctions. Amplitude, Internat. J. Intell. Systems 23:680-714, 2008.

[2] H. Bustince, E. Barrenechea and M. Pagola, J. Fernandez, Interval-valued fuzzy sets constructed from matrices: application to edge detection, Fuzzy Sets and Systems , 160:1819-1840, 2009.

[3] B. De Baets and R. Mesiar, Triangular norms on product lattices, Fuzzy Sets and Systems, 104:61-75, 1999.

[4] G. De Cooman and E. Kerre, Order norms on bounded partially ordered sets, Journal Fuzzy Mathematics, 2:281-310, 1994. .

[5] M. Gehrke, C. Walker and E. Walker, Some comments on interval-valued fuzzy sets, Internat. J. Intell. Systems, 11:751-759, 1996.

[6] J. Goguen, L-Fuzzy Sets, J. Math. Anal. Appli., 18(1):623-668, 1967.

[7] J. Harding, C. Walker and E. Walker, Convex normal functions revisited, Fuzzy Sets and Systems, 161:1343-1349, 2010.

[8] J. Harding, C. Walker and E. Walker, Lattices of convex normal functions, Fuzzy Sets and Systems, 159:1061-1071, 2008.

[9] P. Klement, R. Mesiar and E. Pap, Triangular Norms, Kluwer Academic Publishers, Dordrecht, The Netherlands, 2000.

[10] J. Mendel and R. Jhon, Type-2 fuzzy sets made Simple, IEEE Trans. Fuzzy Systems, 10(2):117-127, 2002.

[11] K. Menger, Statical metrics, Proc. Nat. Acad. Sci. U.S.A., 37:535-537, 1942. 535-537.

[12] M. Mizumoto and K. Tanaka, Fuzzy sets of type-2 under algebraic product and algebraic sum, Fuzzy Sets and Systems, 5: 277-290, 1981.

[13] M. Mizumoto and K. Tanaka, Some properties of fuzzy sets of type-2, Inf. Control, 31:312340, 1976.

[14] S. Ray, Representation of a Boolean algebra by its triangular norms, Matheware and Soft Computing 4:63-68, 1997.

[15] B. Schweizer and A. Sklar, Associative functions and statistical triangle inequalities, Publ. Math., 8:169-186, 1961. 
[16] B. Schweizer and A. Sklar, Statistical metric spaces, Pacific J. Math., 10:313-334, 1960.

[17] C. Walker and E. Walker, Some general comments on fuzzy sets of type-2, Internat. J. Intell. Systems, 24:62-75, 2009.

[18] C. Walker and E. Walker, The algebra of fuzzy truth values, Fuzzy Sets and Systems, 149:309347, 2005.

[19] C. Walker and E. Walker, T-norms for type-2 fuzzy sets, in: Proc. Internat. Conf. on Fuzzy Systems (IEEE 2006), pages 1235-1239, July 16-21, Vancouver (Canadá), 2006.

[20] L. Zadeh, Fuzzy sets, Inf. Control, 20:301-312, 1965.

[21] L. Zadeh, The concept of a linguistic variable and its application to approximate reasoning, Inf. Sci., 8:199-249, 1975. 